\title{
COMPARATIVE INHIBITORY ACTIVITY OF BL-S640 AND TWO OTHER CEPHALOSPORINS
}

\author{
E. Yourassowsky, E. Schoutens and M.P. VANDERLinden \\ Hôpital Universitaire Brugmann, Service de Biologie Clinique, \\ 1020-Bruxelles, Belgique
}

(Received for publication March 20, 1975)

\begin{abstract}
In vitro antibacterial activity of BL-S640 was compared to that of cephalothin and cephalexin against Gram-negative and Gram-positive bacteria isolated from clinical specimens. BL-S640 demonstrated the best activity on nearly all microbial species studied, except for Haemophilus influenzae and Diplococcus pneumoniae against which cephalothin was slightly more active.
\end{abstract}

BL-S640 (a7-(2-aryl-2-aminoacetamido)-3-(heterocyclicthiomethyl) cephalosporin) (Bristol) is a new broad-sepctrum semi-synthetic cephalosporin, which is water-soluble and well absorbed by $\operatorname{man}^{1)}$ and animals after oral administration ${ }^{2)}$.

The present study reports the comparative minimal inhibitory concentrations (MIC) for BL-S640, cephalothin (considered as the leading cephalosporin) and cephalexin (considered as the most representative of the oral cephalosporins) against strains isolated in human pathology.

\section{Material and Methods}

\section{Microbial strains}

The 99 bacteria studied were recently isolated from clinical specimens (urine, blood, pus, sputum, etc.) collected at the Hôpital Universitaire Brugmann, Brussels. Included in the survey were 13 strains each of Salmonella, Proteus, Klebsiella, Escherichia coli, H. influenzae, 8 strains of Listeria monocytogenes, 13 strains of Streptococcus faecalis and 13 strains of D. pneumoniae.

Antibiotic sensitivity testing

MIC were determined by an agar dilution technique, on Mueller-Hinton Agar BBL, to which were added, in the case of $D$. pneumoniae, Haemophilus and St. faecalis, $3 \mu \mathrm{g}$ per $\mathrm{ml}$ of factor V (nicotinamide-adenine dinucleotide phosphate-BDH Chemicals Ltd.) and $2 \%$ defibrinated horse blood. Serial twofold dilutions of the antibiotics (BL-S640, powder for analysis, Bristol; cephalothin and cephalexin, Lilly) were prepared, starting from initial concentrations of 100 $\mu \mathrm{g}$ per ml. Overnight Mueller-Hinton broth cultures were diluted to contain $10^{5}$ organisms per $\mathrm{ml}$ for E. coli, Klebsiella, Listeria, Proteus and Salmonella. D. pneumoniae, Haemophilus and St. faecalis were grown in the same medium supplemented with $0.5 \%$ defibrinated horse blood in a $5 \% \mathrm{CO}_{2}$-enriched atomsphere, and diluted to contain $10^{8}$ organisms per ml. These inocula were spotted with an automatic multipoint inoculator onto the surface of the agar plates ${ }^{3)} . D$. pneumoniae and Haemophilus were incubated in a $5 \% \mathrm{CO}_{2}$-enriched atmosphere.

Results were read after overnight incubation at $37^{\circ} \mathrm{C}$. The MIC was designated as the lowest antibiotic concentration yielding no colony at the site of inoculation.

Disk diffusion tests were performed according to the method described by BAUER et al.4) using the same media as in the MIC determination, and disks containing $30 \mu \mathrm{g}$ of cephalothin, cephalexin or BL-S640.

Inhibition zones were measured with a caliper after overnight incubation at $37^{\circ} \mathrm{C}$. 
Table 1. Activity of BL-S640, cephalothin and cephalexin against Gram-negative bacilli

\begin{tabular}{|c|c|c|c|c|c|c|c|c|c|c|c|c|c|c|}
\hline \multirow{2}{*}{$\begin{array}{l}\text { Microorganisms } \\
\text { (No. of strains) }\end{array}$} & \multirow{2}{*}{ Antibiotic } & \multicolumn{13}{|c|}{ Number of strains inhibited at each concentration $(\mu \mathrm{g} / \mathrm{ml})$} \\
\hline & & 0.048 & 0.097 & 0.195 & 0.39 & 0.78 & 1.56 & 3.12 & 6.25 & 12.5 & 25 & 50 & 100 & $>100$ \\
\hline \multirow{3}{*}{$\begin{array}{c}\text { Salmonella } \\
\text { (13) }\end{array}$} & Cephalothin & 0 & 0 & 0 & 0 & 2 & 11 & 0 & 0 & 0 & 0 & 0 & 0 & 0 \\
\hline & BL-S640 & 0 & 0 & 0 & 11 & 2 & 0 & 0 & 0 & 0 & 0 & 0 & 0 & 0 \\
\hline & Cephalexin & 0 & 0 & 0 & 0 & 0 & 0 & 13 & 0 & 0 & 0 & 0 & 0 & 0 \\
\hline \multirow{3}{*}{$\begin{array}{l}\text { Proteus } \\
\quad(13)\end{array}$} & Cephalothin & 0 & 0 & 0 & 0 & 0 & 2 & 1 & 5 & 1 & 0 & 1 & 3 & 0 \\
\hline & BL-S640 & 0 & 0 & 0 & 2 & 0 & 2 & 4 & 1 & 0 & 3 & 0 & 1 & 0 \\
\hline & Cephalexin & 0 & 0 & 0 & 0 & 0 & 0 & 2 & 3 & 2 & 2 & 2 & 2 & 0 \\
\hline \multirow{3}{*}{$\begin{array}{l}\text { Klebsiella } \\
\text { (13) }\end{array}$} & Cephalothin & 0 & 0 & 0 & 0 & 3 & 1 & 0 & 1 & 3 & 1 & 4 & 0 & 0 \\
\hline & BL-S640 & 0 & 0 & 0 & 3 & 1 & 0 & 3 & 3 & 3 & 0 & 0 & 0 & 0 \\
\hline & Cephalexin & 0 & 0 & 0 & 0 & 0 & 0 & 8 & 4 & 1 & 0 & 0 & 0 & 0 \\
\hline \multirow{3}{*}{$\begin{array}{c}E_{\text {(13) coli }} \\
\text { (13) }\end{array}$} & Cephalothin & 0 & 0 & 0 & 0 & 0 & 5 & 4 & 1 & 2 & 0 & 0 & 0 & 0 \\
\hline & BL-S640 & 0 & 0 & 1 & 5 & 3 & 1 & 0 & 2 & 0 & 0 & 0 & 1 & 0 \\
\hline & Cephalexin & 0 & 0 & 0 & 0 & 0 & 0 & 9 & 1 & 2 & 0 & 0 & 0 & 0 \\
\hline \multirow{3}{*}{$\begin{array}{l}\text { Haemophilus } \\
\text { (13) }\end{array}$} & Cephalothin & 0 & 1 & 3 & 1 & 5 & 2 & 1 & 0 & 0 & 0 & 0 & 0 & 0 \\
\hline & BL-S640 & 0 & 1 & 0 & 4 & 0 & 1 & 7 & 0 & 0 & 0 & 0 & 0 & 0 \\
\hline & Cephalexin & 0 & 0 & 0 & 0 & 0 & 4 & 1 & 2 & 5 & 1 & 0 & 0 & 0 \\
\hline
\end{tabular}

Table 2. Activity of BL-S640, cephalothin and cephalexin against Gram-positive bacteria

\begin{tabular}{|c|c|c|c|c|c|c|c|c|c|c|c|c|c|c|}
\hline \multirow{2}{*}{$\begin{array}{l}\text { Microorganisms } \\
\text { (No. of strains) }\end{array}$} & \multirow{2}{*}{ Antibiotic } & \multicolumn{13}{|c|}{ Number of strains inhibited at each concentration $(\mu \mathrm{g} / \mathrm{ml})$} \\
\hline & & 0.024 & 40.048 & 0.097 & \begin{tabular}{|l|l|}
7 & 0.195 \\
\end{tabular} & 0.39 & 0.78 & 1.56 & 3.12 & 6.25 & 12.5 & 25 & 50 & 100 \\
\hline \multirow{3}{*}{$\begin{array}{l}\text { Listeria } \\
(8)\end{array}$} & Cephalothin & 0 & 0 & 0 & 0 & 0 & 0 & 0 & 8 & 0 & 0 & 0 & 0 & 0 \\
\hline & BL-S640 & 0 & 0 & 0 & 0 & 0 & 0 & 0 & 8 & 0 & 0 & 0 & 0 & 0 \\
\hline & Cephalexin & 0 & 0 & 0 & 0 & 0 & 0 & 0 & 0 & 0 & 0 & 0 & 8 & 0 \\
\hline \multirow{3}{*}{ Str. faecalis } & Cephalothin & 0 & 0 & 0 & 1 & 0 & 0 & 1 & 0 & 0 & 1 & 10 & 0 & 0 \\
\hline & BL-S640 & 0 & 0 & 0 & 0 & 0 & 2 & 0 & 0 & 2 & 9 & 0 & 0 & 0 \\
\hline & Cephalexin & 0 & 0 & 0 & 0 & 0 & 0 & 0 & 1 & 0 & 1 & 0 & 1 & 10 \\
\hline \multirow{3}{*}{ D. pneumoniae } & Cephalothin & 3 & 6 & 4 & 0 & 0 & 0 & 0 & 0 & 0 & 0 & 0 & 0 & 0 \\
\hline & BL-S640 & 1 & 4 & 5 & 3 & 0 & 0 & 0 & 0 & 0 & 0 & 0 & 0 & 0 \\
\hline & Cephalexin & 0 & 0 & 0 & 0 & 1 & 2 & 9 & 1 & 0 & 0 & 0 & 0 & 0 \\
\hline
\end{tabular}

\section{Results}

MIC values obtained for Gram-negative bacilli are shown in Table 1, those for Gram-positive bacteria in Table 2 .

Although there sometimes exist considerable differences between susceptibility of one strain and another amongst the same species, a tentative classification of the 3 drugs, based on the median values $(\mathrm{M})$ of their MIC values was done. Following results were obtained:

Salmonella: BL-S640 $(\mathrm{M}=0.39 \mu \mathrm{g} / \mathrm{ml})$ better than $(>)$ cephalothin $(\mathrm{M}=1.56 \mu \mathrm{g} / \mathrm{ml})>$ cephalexin $(\mathrm{M}=3.12 \mu \mathrm{g} / \mathrm{ml})$.

Proteus: BL-S640 $(\mathrm{M}=6.25 \mu \mathrm{g} / \mathrm{ml})>$ cephalexin $=$ cephalothin $(\mathrm{M}=12.5 \mu \mathrm{g} / \mathrm{ml})$. 
Klebsiella: $\mathrm{BL}-\mathrm{S} 640=$ cephalexin $(\mathrm{M}=3.12$ $\mu \mathrm{g} / \mathrm{ml})>$ cephalothin $(\mathrm{M}=6.25 \mu \mathrm{g} / \mathrm{ml})$.

E. coli: $\quad$ BL-S640 $\quad(\mathrm{M}=0.78 \mu \mathrm{g} / \mathrm{ml})>$ cephalothin=cephalexin $(\mathrm{M}=3.12 \mu \mathrm{g} / \mathrm{ml})$.

H. influenzae: cephalothin $(\mathrm{M}=0.78 \mu \mathrm{g} /$ $\mathrm{ml})>\mathrm{BL}-\mathrm{S} 640 \quad(\mathrm{M}=1.56 \mu \mathrm{g} / \mathrm{ml})>$ cephalexin (M=6.25 $\mu \mathrm{g} / \mathrm{ml})$.

L. monocytogenes: BL-S640=cephalothin $(\mathrm{M}=3.12 \mu \mathrm{g} / \mathrm{ml})>$ cephalexin $(\mathrm{M}=50 \mu \mathrm{g} / \mathrm{ml})$.

St. faecalis: BL-S640 $\quad(\mathrm{M}=6.25 \mu \mathrm{g} / \mathrm{ml})$ $>$ cephalothin $\quad(\mathrm{M}=12.5 \mu \mathrm{g} / \mathrm{ml})>$ cephalexin $(\mathrm{M}=50 \mu \mathrm{g} / \mathrm{ml})$.

Diplococcus pneumoniae: cephalothin $(\mathrm{M}=$ $0.048 \mu \mathrm{g} / \mathrm{ml})>$ BL-S640 $\quad(\mathrm{M}=0.097 \mu \mathrm{g} / \mathrm{ml})>$ cephalexin $(\mathrm{M}=1.56 \mu \mathrm{g} / \mathrm{ml})$.

Thus, in this material, BL-S640 demonstrated the best activity on nearly all of the microbial species studied, except for $H$. influenzae and $D$. pneumoniae against which cephalothin was slightly more active. Its activity was always superior to that of cephalexin except in the case of Klebsiella where it was equivalent.

Fig. 1 illustrates the relationship between zone diameters and MIC values for BL-S640 (Fig. 1a), cephalothin (Fig. 1b) and cephalexin (Fig. 1c).

There was a good agreement between these two methods of sensitivity testing for BL-S640 and cephalothin. Only St. faecalis appeared slightly more sensitive by the disk method than by the agar diffusion technique; the zone diameters remain nonetheless grouped within the intermediate sensitivity zone.

Cephalexin, on the other hand, did not demonstrate as fine a distinction between sensitive and resistant strains with the disk method, as did the other two antibiotics: several strains were found in the intermediate sensitivity zone. However, the poor activity of cephalexin on St. faecalis, as illustrated by a mean MIC value of $50 \mu \mathrm{g} / \mathrm{ml}$, was confirmed by the disk method: 11 of the 13 strains had zone diameters inferior to the critical diameter of $14 \mathrm{~mm}$.
Fig. 1. Relationship between zone diameters (30$\mu \mathrm{g}$ disks) and MIC values for BL-S640 (Fig. 1a), cephalothin (Fig. 1b) and cephalexin (Fig. 1c)

(a)

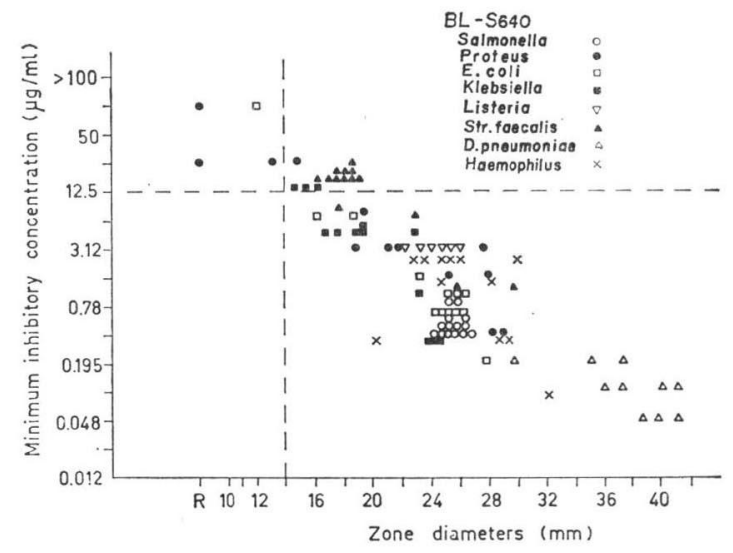

(b)

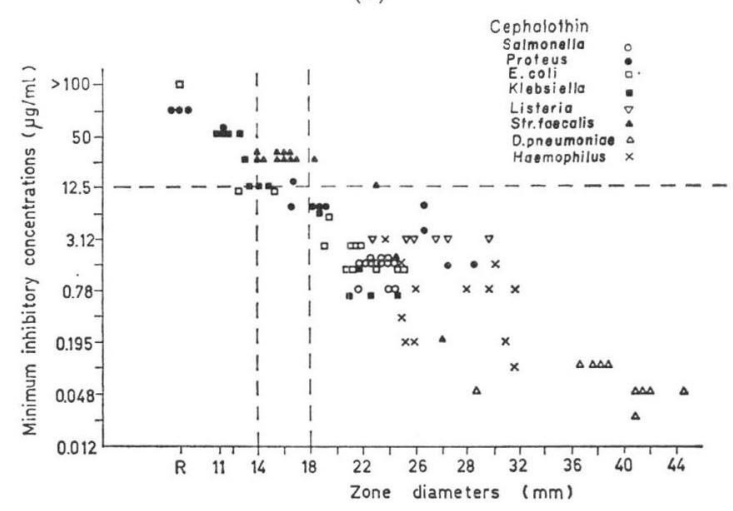

(c)

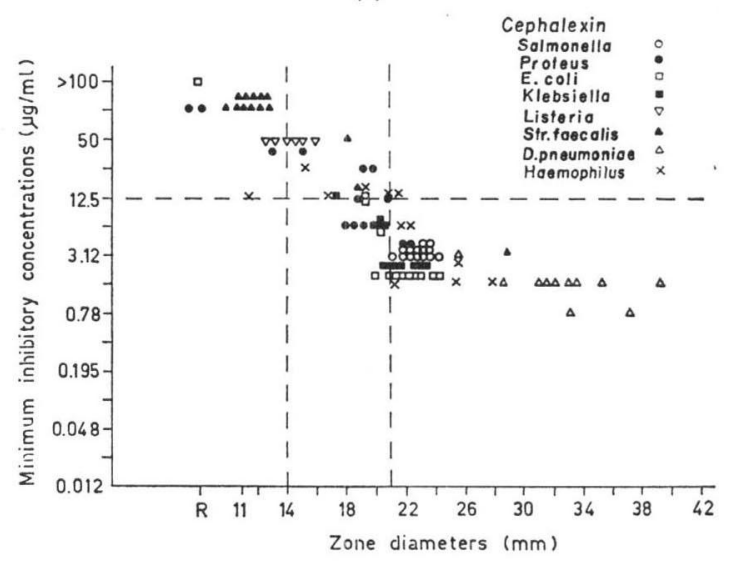




\section{Discussion and Conclusions}

BL-S640 is an oral cephalosporin presenting an in vitro antibacterial activity comparable to that of cephalothin (considered as the first choice among the cephalosporins) and considerably superior to that of cephalexin.

BL-S640 could be a valuable drug among the oral cephalosporins: it has been shown (in animal experiments) that peak blood concentrations of this drug are similar to those obtained with cephalexin when administered at the same oral $\operatorname{doses}^{2}$, but blood levels are more prolonged. Moreover, volume of tissue distribution of this drug increases as dosage increases ${ }^{1)}$. Clinical efficacy in man, as well as toxicity and side-effects of BL-S640 must be further investigated.

\section{References}

1) Pfeffer, M.; D. L. Hudson \& D. R. Van Harken: Dose-dependent human pharmacokinetics of BL-S640. 14th Intersci. Conf. Antimicr. Agents \& Chemoth. Abstract paper No. 422. San Francisco 1974

2) Leitner, F.; R.E. Buck, M. Misiek, T.A. Pursiano, D. R. Chisholm, Y.H. Tsai, G. E. Wright, \& K. E. PRICE: BL-S640, a new broad spectrum cephalosporin. 14th Intersci. Conf. Antimicr. Agents \& Chemoth. Abstract paper No. 421. San Francisco 1974

3) Steers, E.; E.L. Foltz \& B. S. Graves: An inocula replicating apparatus for routine testing of bacterial susceptibility to antibiotics. Antibiot. \& Chemoth. 9: 307 311, 1959

4) Bauer A. W.; W. M. M. Kirby, J. C. Sherris \& M. Turk: Antibiotic susceptibility testing by a standardized single disk method. Amer. J. Clin. Pathol. 45: 493 496, 1966 